\title{
Transients Due to Multiple Prestrike Phenome- non when Energizing Capacitor Banks with a Vacuum Circuit-Breaker
}

\author{
M. B. Barbieri, IEEE Senior Member(*), R. E. Bianchi Lastra, IEEE Senior Member(*), \\ P. L. Arnera IEEE Senior Member (*), and J. L. Agüero IEEE Senior Member (*),
}

\begin{abstract}
The phenomenon of multiple prestrikes of the arc was observed in field measurements of the energization of a single 4.8 MVAR, $13.2 \mathrm{kV}$ capacitor bank. This paper presents field measurements records and the results of the computer simulation of the transients when energizing the capacitor bank, made with the objective to calculate the overvoltages generated in remote points. A method to avoid the phenomenon is proposed.
\end{abstract}

Index Terms-Capacitor Switching, Multiple Prestrikes, Vacuum Circuit Breakers.

\section{INTRODUCTION}

T $\mathrm{n}$ utilities it is common the use of shunt capacitor banks to I provide voltage support due to the lack of an adequate power transmission system. The size and location of this capacitor banks are usually based on load flow studies, and sometimes the necessary amount of MVAR must be divided in two or more modules in the same busbar, to provide a better regulation of the bus voltage.

The energization of a capacitor bank, when one or more banks are already in service in the same bus (called 'Back-toBack' capacitor switching), is one of the cases when the main transient is the overcurrent that flows between the circuit breaker and the capacitor banks, and not the overvoltages in the buses.

This current between circuit breaker and capacitor banks is only limited by stray inductances in the circuit, and if countermeasures are not taken, this current can be of high amplitude and/or frequency. To avoid damage of circuit breakers and/or capacitors, the standards impose limits to the amplitude and frequency of this current, and it is generally necessary to insert an inductance in series with the capacitor banks to meet the requirements of the standards.

In other cases, a single capacitor bank is installed on the bus because it is enough to provide the adequate voltage support. In this situation the insertion current is normally limited by the inductance of the source, and then no limited inductance is normally necessary.

However, an interesting case is when in the same bus where the single capacitor bank (without a limiting induc-

(*) M. B. Barbieri, R. E. Bianchi Lastra, P. L. Arnera, and J.L. Agüero work in IITREE-LAT. Facultad de Ingeniería Universidad Nacional de La Plata. (1900) 48 y 116. La Plata. Argentina (e-mail: iitree@iitree-unlp.org.ar). tance) is, there are also connected several cables, overhead lines or both combined, (together with stray capacitances of other equipments on the bus). In this situation, the equivalent capacitances of these elements work as a 'small capacitor bank' and then the insertion current when the single bank is energized, can have similar characteristics of Back-to-Back energization, and a current component of high amplitude and frequency flow between the circuit breaker and capacitor.

\section{A. The multiple prestrike phenomenon.}

Practically in all cases, when a capacitor bank is energized, the arc current is established in the circuit breaker a short time before the mechanical contact closure.

This phenomenon, named 'prestrike', is very complex and difficult to predict. The currents and overvoltages that are produced depend on many factors, like circuit-breaker characteristics, dielectric properties, surge impedance of the involved elements, high frequency current interruption capability and pole scatter and point of wave of closing.

The prestrike produces current and voltage waves that flow by cable/lines to the load where reflection takes place. The reflected waves return to the breaker terminals, and the current that flows as an arc across the closing contact gap can be then of high frequency. The prestriking arc may then be interrupted at or near a current zero, depending on the rate of change of current as it passes through the current zero.

If interruption does occur, the dielectric strength will recover until the voltage across the contacts overcomes again the dielectric strength of the decreasing gap. The process may be repeated several times until the contacts touch, and numerous high frequency current zeros can occur.

SF6 circuit-breakers are generally unable to interrupt high frequency current, and usually only a single prestrike transient occurs. However, some authors [1] agree that few prestrikes (one or two) may occur on closing a SF6 contactor under certain conditions.

Vacuum circuit breaker, however, has the ability of interrupting high frequency currents in each crossing through zero, so it is possible to find many prestrikes in the short interval between the prestrike of the arc and the mechanical closure of the contacts. This phenomenon is named 'multiple prestrikes', and the number of prestrikes depend on the interruptible $\mathrm{di} / \mathrm{dt}$ of the vacuum circuit-breaker, on the rise rate of the voltage across the contacts when the current is interrupted, and on the 